\title{
OBSERVATION DU GRAPHITE EN MICROSCOPIE ÉLECTRONIQUE A BALAYAGE EN TRANSMISSION
}

\author{
P. GUYOT \\ LTPCM, LA no 29, ENSEEG, Domaine Universitaire, B. P. 44, 38401 St-Martin-d'Hérès, France \\ et
}

\section{J. M. GJURASEVIC}

Pechiney, Centre de Recherches, 38340 Voreppe, France

(Reçu le 8 juillet 1975, révisé le 5 novembre 1975, accepté le 18 novembre 1975)

\begin{abstract}
Résumé. - Un microscope électronique à balayage conventionnel fonctionnant à $30 \mathrm{kV}$ en émission thermo-ionique est utilisé pour observer principalement en transmission les dislocations dans le graphite. On étudie l'influence des angles d'illumination et de collection sur les images. Les contrastes observés sont convenablement expliqués à partir du théorème de réciprocité.

Abstract. - A conventional scanning electron microscope working at $30 \mathrm{kV}$ with thermo-ionic emission and principally in the transmission mode is used to observe the dislocations in graphite. The influence of the illumination and collecting angles on the image is studied. The observed contrasts are conveniently explained in terms of the reciprocity theorem.
\end{abstract}

1. Introduction. - La microscopie électronique à balayage en transmission (M. E. B. T.) présente certains avantages par rapport à la microscopie électronique à transmission conventionnelle (M. E. T. C.) :

- Possibilité de traitement du signal en chaque point de l'image.

- Faible irradiation aux électrons de l'échantillon.

- Pénétration plus grande, au moins pour les éléments légers [1], qu'en M. E. T. C., où l'élargissement du faisceau d'électrons et les pertes d'énergie dans l'échantillon entraînent, par suite des aberrations sphériques et chromatiques de l'objectif, une détérioration de la résolution : cf. par exemple l'observation comparée de lames de plusieurs $\mu \mathrm{m}$ d'échantillons biologiques à $100 \mathrm{kV}$ [14], de $1,1 \mu \mathrm{m}$ de $\mathrm{Cu}-10 \% \mathrm{Al}$ à $200 \mathrm{kV}$ [15].

- Possibilité de détection simultanée de différents signaux, comme par exemple celle des électrons inélastiques aux petits angles et des électrons élastiques aux grands angles permettant d'obtenir un meilleur contraste [2].

En ce qui concerne les contrastes obtenus en M. E. T. C. et M. E. B. T., leur similarité est expliquée par le théorème de réciprocité $[3,4]$, qui fait état de l'équivalence entre M. E. T. C. et M. E. B. T. dans la mesure où source d'illumination et détecteur sont inversés. Cette équivalence n'est complète que si les conditions de fonctionnement des deux appareils (excitation des lentilles, diaphragmes) sont identiques, ce qui n'est en général pas le cas : si les angles correspondants, ouverture de l'objectif $\alpha_{C}$ en M. E. T. C. et angle d'illumination $\alpha_{B}$ en M. E. B. T., peuvent être rendus du même ordre de grandeur afin de minimiser les aberrations sphériques des objectifs respectifs, il n'en est plus de même pour l'angle d'illumination $\beta_{\mathrm{C}}$ en M. E. T. C. et de collection $\beta_{\mathrm{B}}$ en M. E. B. T. : $\beta_{\mathrm{B}}$ est conditionné par l'obtention d'un rapport signal sur bruit aussi grand que possible, si bien que $\beta_{\mathbf{B}} \gg \beta_{\mathrm{C}}$. Des différences de contraste entre M.E.T.C. et M. E. B. T. sont alors nécessairement observées : détérioration des diagrammes de diffraction quand $\beta_{\mathbf{B}}$ augmente [5], disparition des effets dynamiques [6, 7], qui s'interprètent facilement en théorie du contraste en considérant la non-collimation du faisceau incident.

L'observation des dislocations en M. E. B. T. impose une résolution spatiale donc un diamètre de la sonde électronique inférieur ou égal à la largeur des images $\Delta \chi \sim \xi_{g} / 3$ pour les réflexions $\bar{g}$ de distance d'extinction $\xi_{g}$ telles que $\bar{g} \cdot \vec{b}=1$. Les dislocations ont par suite été résolues en M. E. B. T. :

i) Dans des matériaux à $\xi_{g}$ élevée : $\mathrm{HfS}_{2}, \mathrm{SnS}_{2}$, $\mathrm{MoS}_{2}[6,7,8]$.

ii) Dans des métaux à relativement faible $\xi_{g}$, tels $\mathrm{Al}$ et $\mathrm{Si}$, avec des canons à forte brillance : tensions d'accélération de 80 à $100 \mathrm{kV}$, filaments $\mathrm{LaB}_{6}$ ou à pointe $[9$, 10], émission de champ. 
Dans un microscope conventionnel, fonctionnant en émission thermo-ionique classique sous une tension d'accélération de $30 \mathrm{kV}$, la difficulté est double : faible brillance du canon par ailleurs proportionnelle à la tension d'accélération, faible $\xi_{y}$ (proportionnelle à l'inverse de la longueur d'onde électronique : $\xi_{g} \sim 300 \AA$ pour les réflexions de bas indices de l'aluminium et du silicium à $30 \mathrm{kV}$ ). Nous avons néanmoins réalisé ces observations dans l'aluminium [5] et le silicium, sans modifier le système classique de détection et grâce à une amplification intermédiaire de courant placée entre l'échantillon et le détecteur. Le graphite, dont $\xi_{10 \overline{1} 0}=1200 \AA$ à $30 \mathrm{kV}$, pose par contre relativement moins de problèmes. Nous en rapportons ici l'observation des dislocations.

2. Observation en M. E. B. des dislocations dans le graphite. - Le microscope utilisé est un Cambridge-S $430 \mathrm{kV}$. Le porte-objet en transmission a été décrit par ailleurs [5]; dans sa version actuelle le diaphragme de collection est fixe par rapport à la platine et ne permet pas de réaliser des champs noirs sélectifs. Les électrons transmis créent des électrons secondaires dans une feuille d'aluminium ou de platine, placée sous l'échantillon, avec un taux de conversion voisin de 1,3. Les électrons secondaires sont ensuite collectés par le détecteur scintillateur-photomultiplicateur standard. Les conditions d'observation ont été améliorées par rapport au précédent travail [5] sur l'aluminium par l'utilisation d'un filament de tungstène à pointe.

Le graphite hexagonal est un empilement hexagonal compact ...abab... de plans où les atomes de carbone sont situés aux nœuds d'un réseau hexagonal. Avec les notations habituelles les dislocations dans le plan de base ont des vecteurs de Burgers du type AB ( $\left.\frac{1}{3}[11 \overline{2} 0]\right)$ pour les parfaites, du type $\mathbf{A} \boldsymbol{\alpha}$ ou $\mathbf{A \beta}\left(\frac{1}{3}[10 \overline{10}]\right)$ pour les partielles. Elles ont été observées en M. E. T. C. à $100 \mathrm{kV}$ par Amelinckx et Delavignette [11] ; l'énergie $\gamma$ de faute d'empilement étant de $\sim 2,5 \times 10^{-2} \mathrm{erg} / \mathrm{cm}^{2}$ les dissociations sont supérieures à quelques $100 \AA$ et donc facilement observées.

Les lames minces, obtenues par clivages successifs, sont parallèles au plan de base. Leur épaisseur, déterminée par stéréoscopie, est d'environ $1500 \AA$.

DiAgRAMme DE DIFFRACTION. - Le diagramme de diffraction d'axe de zone [0001], représenté sur la figure 1, a été obtenu sans diaphragme objectif et en abaissant le point de rotation du faisceau sur la surface même de l'échantillon. La résolution angulaire des taches, en accord avec le théorème de réciprocité, est d'autant meilleure que l'angle de collection $\beta_{\mathbf{B}}$ est petit.

Dislocations. - Nous avons d'abord constaté, en accord avec la référence [6] une diminution du contraste des contours d'extinction et des dislocations à angle de collection croissant : en faisant varier $\beta_{\mathbf{B}}$ de 3 à $62 \mathrm{mrad}$ pour $\alpha_{\mathrm{B}}=50 \mathrm{mrad}$, on observe une stabili-

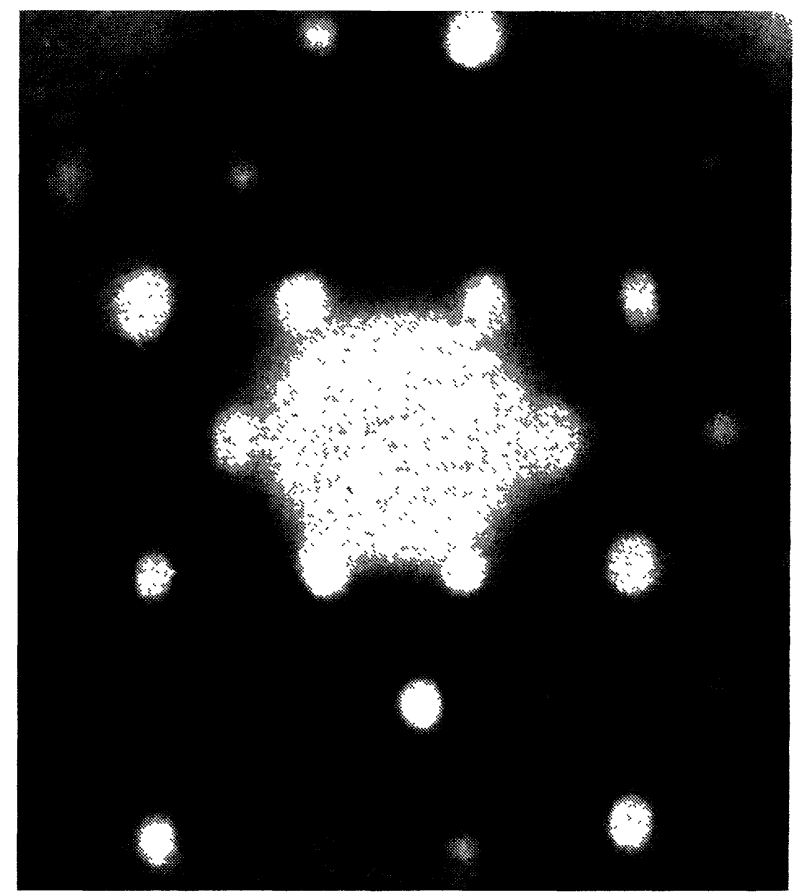

Fig. 1. - Diagramme de diffraction $\beta_{\mathrm{B}}=3 \times 10^{-3} \mathrm{rad}$. Axe de zone : 0001 .

sation du contraste au-delà de $\beta_{\mathrm{B}} \sim 30 \mathrm{mrad}$. Cet effet est interprété [7], [12] à partir du théorème de réciprocité, pour un effet de moyenne sur la convergence $2 \beta_{\mathrm{C}}$ en M. E. T. C., soit $2 \beta_{\text {B }}$ en M. E. B. T. Quand $\beta_{\mathrm{B}}>\theta_{\mathrm{B}}$, angle de Bragg pour la réflexion considérée (présentement $16,5 \mathrm{mrad}$ pour $\bar{g}=10 \overline{10}$ ) le champ noir vient se superposer au champ clair. Il en résulte un gain d'intensité collectée, mais certains contrastes devraient en principe disparaître, comme pour les dislocations situées près de la face de sortie de la lame dont les images en champ clair et champ noir, par suite des phénomènes d'absorption, sont alors complémentaires.

Les figures suivantes illustrent l'observation des dislocations :

- les micrographies $2 a$ et $2 b$ diffèrent par l'angle d'illumination $\alpha_{B}$. L'augmentation de $\alpha_{B}$ augmente le diamètre et le courant du faisceau au niveau de l'échantillon. Effectivement, le bruit de fond est plus faible pour la figure $2 a$ que pour la figure $2 b$, mais la résolution y est moins bonne.

- Les micrographies $2 c$ et $2 d$ diffèrent par l'angle de collection; les deux angles $\beta_{\mathrm{B}}$ étant supérieurs à l'angle de Bragg le contraste des dislocations évolue peu. Des dissociations doubles (D) et triples symétriques (TS) sont observées. Bien que notre platine porte-objet ne permette pas de se placer strictement à deux ondes et donc de déterminer les vecteurs de Burgers, ces dissociations semblent bien correspondre à celles décrites dans [11] : pour (D), $\mathbf{A B}=\mathbf{A} \boldsymbol{\alpha}+\boldsymbol{\alpha} \mathbf{B}$, la largeur de dissociation varie entre quelques $100 \AA$ et $1000 \AA$ suivant le caractère des dislocations, en accord avec la valeur de $\gamma$; la largeur de dissociation de (TS), composée de 3 dislocations du type $\mathbf{A} \alpha$, est d'environ 5 fois 


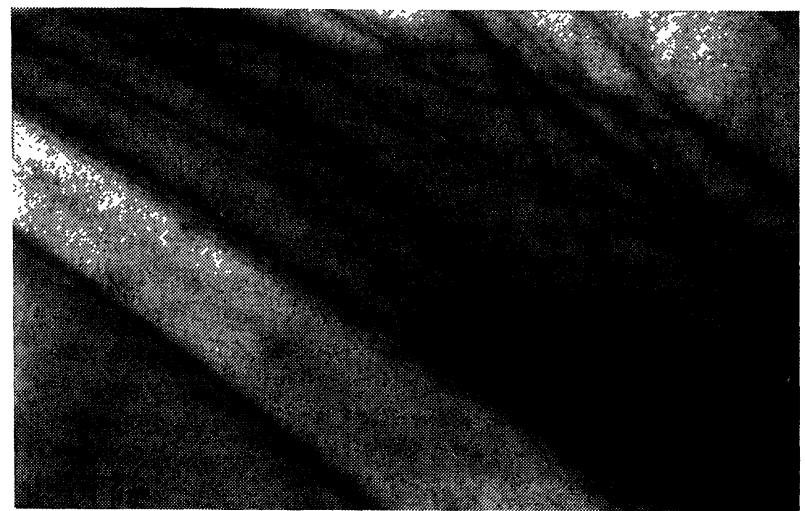

(a)

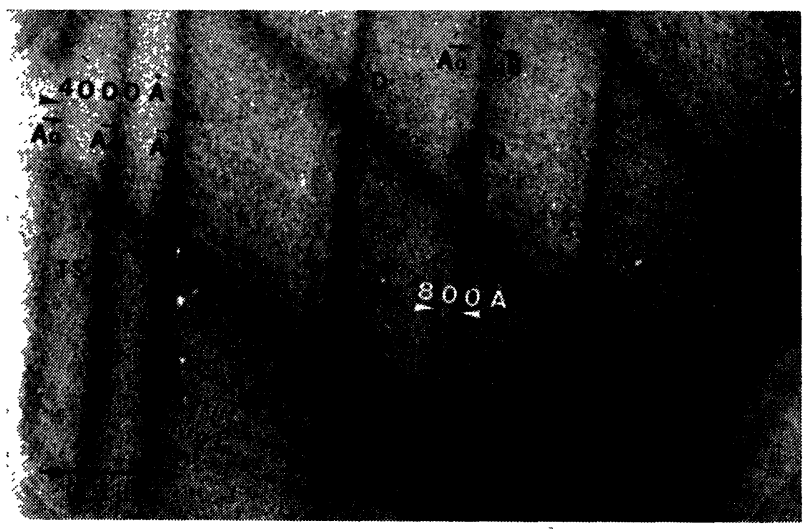

(c)

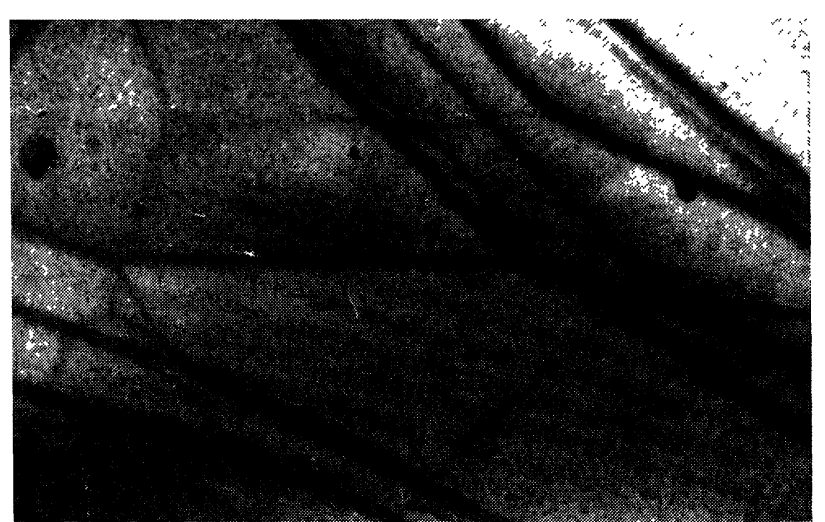

(b)

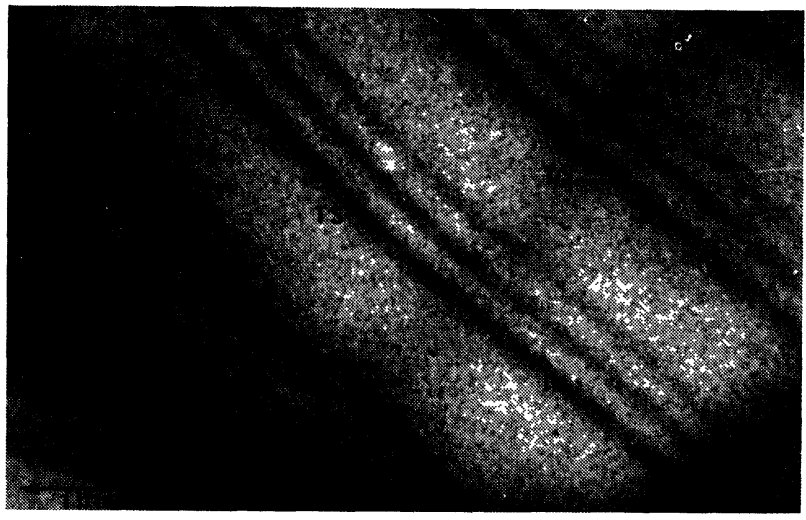

(d)

Fig. 2. - Dislocations dissociées dans le plan de base.

a) $\alpha_{\mathrm{B}}=5 \quad \times 10^{-2} \mathrm{rad}, \quad \beta_{\mathrm{B}}=0,62 \times 10^{-1} \mathrm{rad}$,

b) $\alpha_{\mathrm{B}}=1,25 \times 10^{-2} \mathrm{rad}, \quad \beta_{\mathrm{B}}=0,62 \times 10^{-1} \mathrm{rad}$,

c) $\alpha_{\mathrm{B}}=1,25 \times 10^{-2} \mathrm{rad}, \beta_{\mathrm{B}}=1,85 \times 10^{-2} \mathrm{rad}$,

d) $\alpha_{\mathrm{B}}=1,25 \times 10^{-2} \mathrm{rad}, \beta_{\mathrm{B}}=0,62 \times 10^{-1} \mathrm{rad}$.

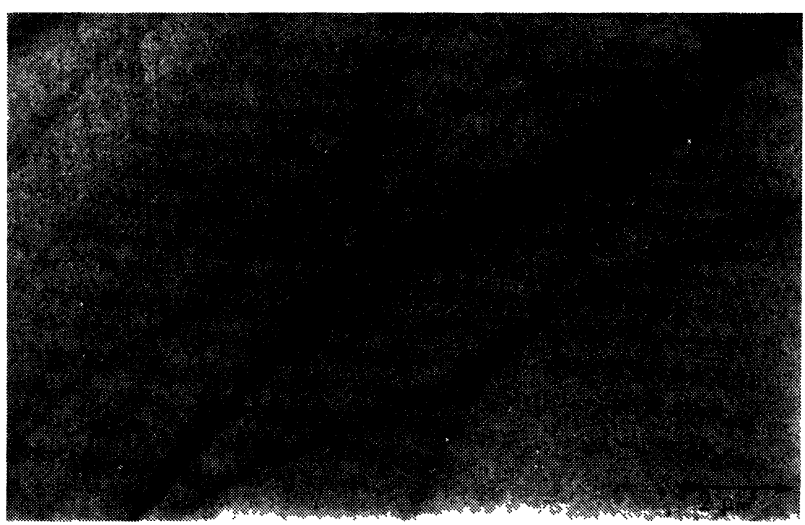

(a)

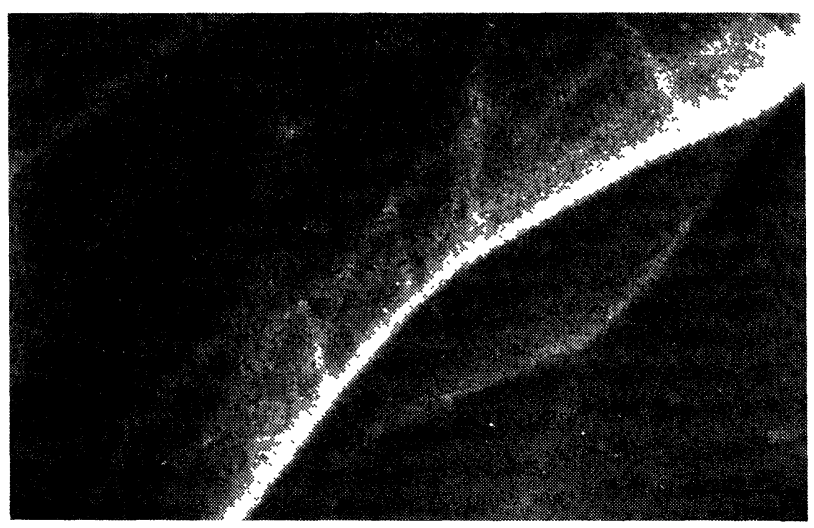

(b)

FIG. 3. '- Dislocations dans le plan de base. Images en $a$ ) transmission, $b$ ) réflexion.

plus large que (D) à caractère identique, en accord avec les calculs d'équilibre des trois partielles.

- Enfin les micrographies $3 a$ et $3 b$ représentent la même plage, en transmission et en réflexion. Les clichés ont été réalisés avec un filament de tungstène standard en épingle à cheveux; la faible brillance du canon explique (cf. par exemple [13]) la mauvaise résolution en réflexion: les dissociations ne sont plus résolues.

3. Conclusion. - Il est possible malgré la faible brillance de résoudre, au prix de modifications réduites, les défauts cristallins par transmission, voire par réfle- 
xion, dans un microscope à balayage conventionnel fonctionnant avec une tension d'accélération de $30 \mathrm{kV}$ en émission thermo-ionique. L'interprétation des contrastes est donnée par le théorème de réciprocité et la théorie classique du contraste. On en déduit ainsi que l'utilisation d'un petit diaphragme de collection
$\left(\beta_{\mathbf{B}} \sim\right.$ quelques $\left.10^{-3} \mathrm{rad}\right)$ permet l'obention de bons diagrammes de diffraction et préserve les contrastes dynamiques des défauts, et qu'une faible convergence du faisceau incident $\left(\alpha_{B} \sim 10^{-2} \mathrm{rad}\right)$ améliore la résolution dans les limites d'un rapport signal sur bruit de fond convenable.

\section{Bibliographie}

[1] Fraser, H. L. and Jones, I. P., Phil. Mag. 31 (1975) 225.

[2] Crewe, A. V., Wall, J., Septième Congrès International de Microscopie Electronique, Grenoble 1 (1970) 35.

[3] Cowley, J. M., Appl. Phys. Lett. 15 (1969) 58.

[4] HowIE, A., Proc. fifth European Congress on Electron Microscopy (1972) 408.

[5] Guyot, P., Guurasevic, J. M., Z. Naturforsch. 29a (1974) 1381.

[6] Graf von Harrach, H., Walker, A. R. and Joy, D. C., Scanning electron microscopy : Systems and applications 1973. Conference Series number 18. The Institute of Physics, 170-175.

[7] Booker, G. R., Joy, D. C., Spencer, J. P. and von HarRACH, H., Eight International Congress on Electron Microscopy, Canberra 1 (1974) 284.
[8] Stern, R. M., Revue Phys. Appl. 9 (1974) 377.

[9] Humphreys, C. J., Spencer, J. P., Woolf, R. J., Joy, D. C., Titchmarsh, J. M. and Booker, G. R., Proceedings of the fifth annual Scanning Electron Microscope Symposium, Chicago (1972) 205.

[10] Clarke, D. R., Phil. Mag. 24 (1971) 973.

[11] Amelinckx, S. and Delavignette, P., Electron Microscopy and strength of crystals (Ed. G. Thomas, J. WashburnInterscience) 1963, p. 441.

[12] Melander, A. and Sandström, R., Phys. Stat. Sol. 22 (1974) 587.

[13] Guyot, P., Revue Phys. Appl. 9 (1974) 393.

[14] Kolke, H., Matsuo, T., Ueno, K. and Suzuki, M., JEOL News 10 (1972) 6.

[15] Yamamoto, T., Nishizawa, H., JEOL News 12 (1974) 19. 